\title{
Coded-Aperture Imaging Using Photo-induced Reconfigurable Aperture Arrays for Mapping Terahertz Beams
}

\author{
Akash Kannegulla ${ }^{1}$, Zhenguo Jiang ${ }^{1}$, Syed Rahman ${ }^{1}$, Patrick Fay ${ }^{1}$, Huili Grace Xing ${ }^{1}$, Li-Jing \\ Cheng $^{2 *}$, Lei Liu ${ }^{*}$ \\ ${ }^{1}$ Department of Electrical Engineering, University of Notre Dame, Notre Dame, IN 46556, \\ USA.Email: 1liu3@nd.edu \\ ${ }^{2}$ School of Electrical Engineering and Computer Science, Oregon State University, OR \\ 97311, USA. Email: chengli@eecs.oregonstate.edu
}

\begin{abstract}
We report terahertz coded-aperture imaging using photo-induced reconfigurable aperture arrays on a silicon wafer. The coded aperture was implemented using programmable illumination from a commercially available digital light processing projector. At $590 \mathrm{GHz}$, each of the array element apertures can be optically turned on and off with a modulation depth of $20 \mathrm{~dB}$ and a modulation rate of $\sim 1.3 \mathrm{kHz}$. Prototype demonstrations of $4 \times 4$ codedaperture imaging using Hadamard coding have been performed and this technique has been successfully applied to mapping $\mathrm{THz}$ beams by using a $6 \times 6$ aperture array at $590 \mathrm{GHz}$. The imaging results agree closely with theoretical calculations based on Gaussian beam transformation, demonstrating that this technique is promising for realizing real-time and low-cost terahertz cameras for many applications. The reported approach provides a simple but powerful means to visualize $\mathrm{THz}$ beams, which is highly desired in quasi-optical system alignment, quantum-cascade laser design and characterization, and $\mathrm{THz}$ antenna characterization.
\end{abstract}


The submillimeter-wave and terahertz $(\mathrm{THz})$ region in the electromagnetic spectrum has become more and more important to radio astronomy, chemical spectroscopy, bio-sensing, medical imaging, security screening and defense [1-4]. In recent years, technologists have intensified their efforts to develop imaging systems operating in the $\mathrm{THz}$ region for all the above applications [5-8]. In addition, high-performance and low-cost imaging devices are highly desired for $\mathrm{THz}$ beam visualization for scientific metrology applications such as quasioptical system alignment, quantum-cascade laser (QCL) design and optimization [9], and $\mathrm{THz}$ antenna characterization [10]. To date, the $\mathrm{THz}$ imaging systems that have been demonstrated generally fall into one of three categories: (1) single-element imagers that obtain images by mechanical scanning, (2) array imagers (e.g. focal-plane arrays (FPAs)) that consist of an array of imaging sensor elements [6-8], and (3) coded-aperture imaging (CAI) using two-dimensional aperture masks [11-14]. In many applications, important events happen on the scale of microseconds, making imaging by mechanical scanning impractical due to the inherently low frame rates. Array-based imagers such as FPAs can greatly reduce observing and processing time by recording imaging information in parallel. Although FPAs offer the highest imaging speed and signal-to-noise ratio, they tend to be complicated and expensive, especially for large-scale arrays with high imaging resolution.

Compared to $\mathrm{THz}$ imaging using mechanical scanning and focal-plane arrays, CAI offers the advantage of both high performance (i.e., high signal-to-noise ratio and high frame rate) as well as the potential for realizing simple and low-cost systems. CAI-based systems are based on spatial encoding and modulation to eliminate the need for detector arrays. In this imaging technique, a single $\mathrm{THz}$ detector in combination with a series of $N \mathrm{x} N$ coded aperture masks is required in order to obtain an image with an $N$ x $N$ resolution. Measurements with $N^{2}$ masks are taken, and the same number of linear equations are then solved to reconstruct the object image [11]. This basic concept has been demonstrated using masks fabricated on 
printed circuit boards [12]. In order to achieve high frame rates with $\mathrm{THz} \mathrm{CAI}$, aperture arrays electronically actuated by Schottky diodes [13] and graphene modulators [14], respectively, have been proposed to realize the required coded masking. However, these approaches require complicated and prepatterned circuits for operation, which also results in expensive and complex systems.

In this letter, we report $\mathrm{THz} \mathrm{CAI}$ using photo-induced reconfigurable aperture arrays using an unpatterned silicon wafer illuminated by a commercially available digital light processing projector (DLP). The optical THz modulation mechanism described in [15] was employed to spatially modulate each array pixel. Pixels illuminated with light will be turned "off" due to increased photo-induced free carriers and local conductivity, while other pixels remain highly transparent ("on") to THz signal. This approach allows us to generate extremely large-scale reconfigurable coded masks (e.g., $1024 \times 768$, only limited by the DLP resolution and carrier diffusion lengths in the $\mathrm{Si}$ wafer) for $\mathrm{THz} \mathrm{CAI}$, without the need for any microfabrication processes or precise alignments. We demonstrate that at $590 \mathrm{GHz}$, each array element aperture (pixel) can be effectively turned "on" and "off" with a modulation depth of $20 \mathrm{~dB}$ and at a modulation rate of $\sim 1.3 \mathrm{kHz}$. Prototype demonstrations of $4 \times 4$ coded-aperture imaging using Hadamard coding [11] have been performed and this technique has been successfully applied to mapping THz beams with $6 \times 6$ pixels at $590 \mathrm{GHz}$.

Fig. 1 shows the experimental setup $[15,16]$ in which a frequency multiplication chain based on Schottky diode multipliers (Virginia Diodes, Inc.) was used as a $\mathrm{THz}$ source in the frequency range of $570-600 \mathrm{GHz}$ with an average output power of approximately $1 \mathrm{~mW}$. The output was coupled to free space using a WR-1.5 horn antenna. Four off-axis parabolic mirrors (M1-M4 in Fig. 1) were used to collimate and focus the THz beam. After passing through the Si wafer that serves as the coded aperture, the focused $\mathrm{THz}$ beam was reflected by an indium tin oxide (ITO) coated glass plate onto a broadband zero-bias Schottky diode 


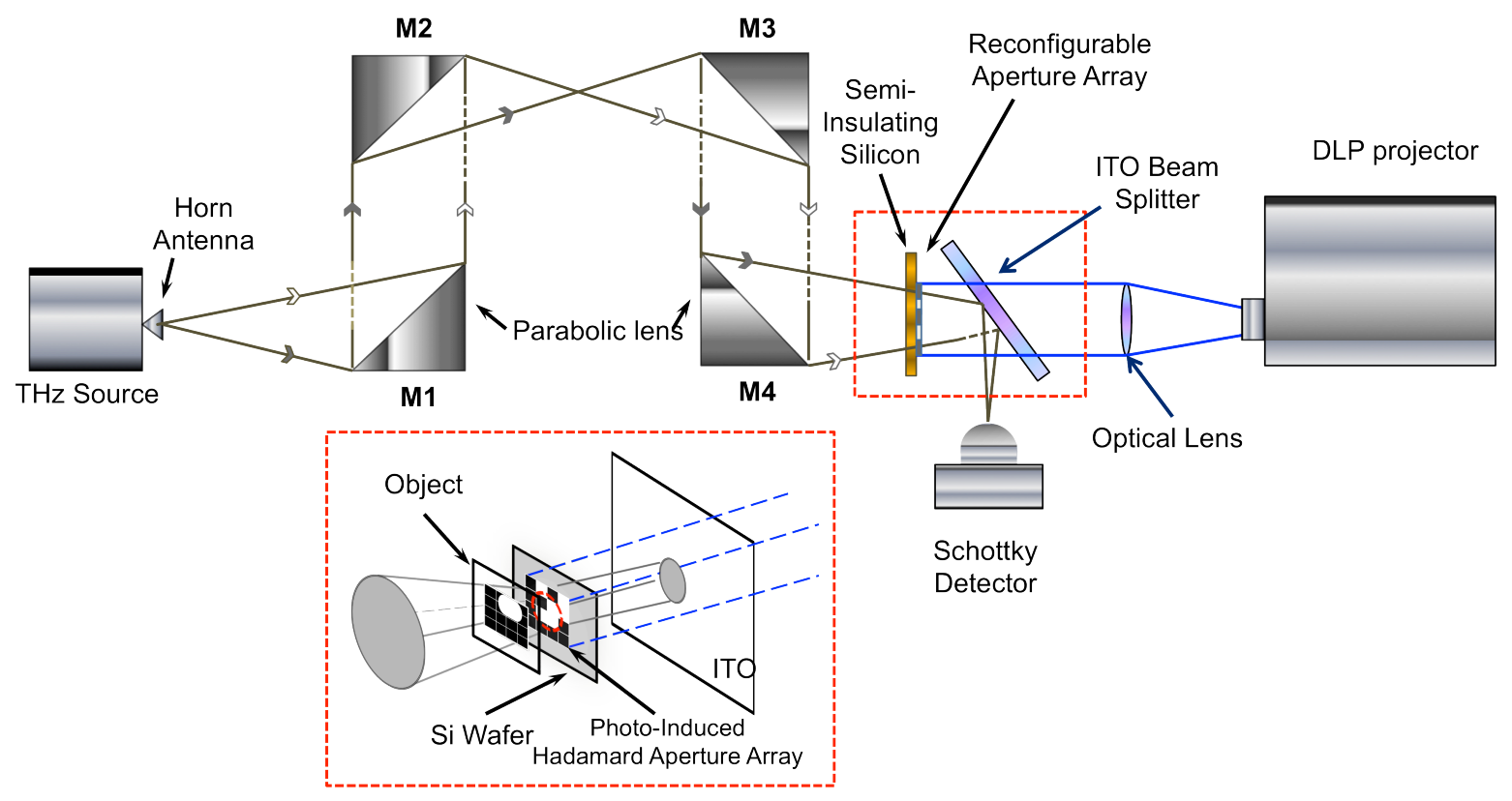

Fig.1. (color online) Diagram of the experiment setup. Reconfigurable photo-induced Hadamard aperture array was produced on a semi-insulating silicon wafer for $\mathrm{THz}$ coded-aperture imaging at $590 \mathrm{GHz}$. A commercially available DLP project was employed for generating the Hadamard patterns.

(ZBD) detector [17]. The ITO-coated plate was mounted at $45^{\circ}$ with respect to the $\mathrm{THz}$ beam; the ITO-coated plate reflects the $\mathrm{THz}$ signal, but is optically transparent so that the optical pattern from the DLP can project onto the Si wafer. A $200 \mu \mathrm{m}$ thick double-side polished semi-insulating silicon wafer was used as the coded-aperture modulator. This silicon wafer was illuminated by a commercially available DLP to generate reconfigurable Hadamard coded aperture arrays for CAI (see Fig. 1 inset for a representative $4 \times 4$ Hadamard array). The DLP system consisted of a 0.7 " digital mirror device (DMD) panel with $1024 \times 768$ dot resolution and a $200 \mathrm{~W}$ mercury lamp. For this application, the RGB filter wheel was removed to allow generating white light images with a maximum brightness of $\sim 2500$ lumens after passing through a visible bandpass filter $(400-800 \mathrm{~nm})$. To focus the projected image down to an area of $10 \times 10 \mathrm{~mm}^{2}$ on the Si wafer, an additional lens was inserted in the optical path. For the imaging demonstration, a thin metal target (covered by 
absorber) with an open aperture was placed in the $\mathrm{THz}$ beam just before the silicon wafer (position "O" in the inset of Fig. 1) to serve as an object. For the THz beam mapping application, the silicon wafer was moved axially along the $\mathrm{THz}$ beam while keeping the aperture array patterns focused (the DLP moved together with the silicon wafer).

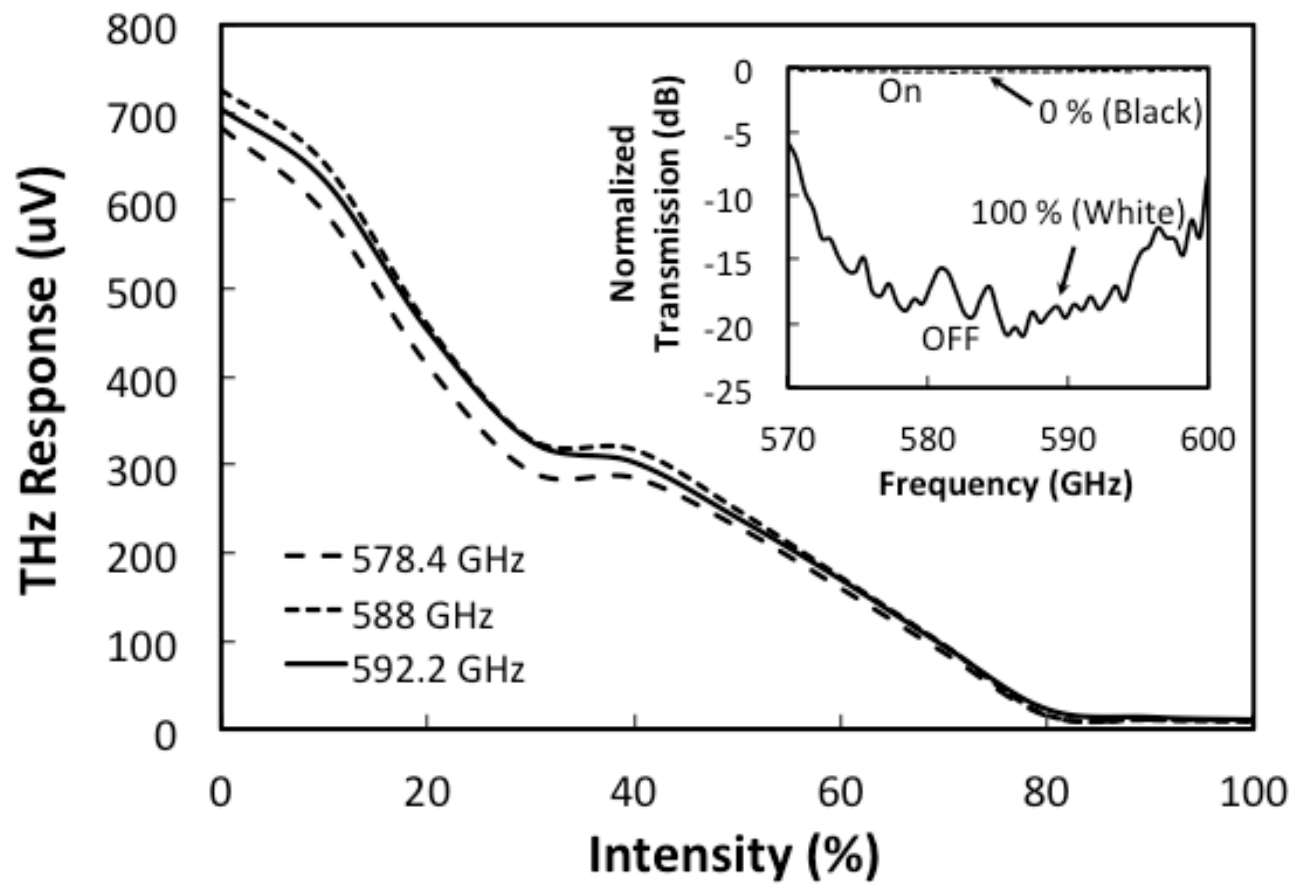

Fig. 2: (a) Measured THz responses from the detector as a function of DLP light intensity at three frequencies, i.e., $578.4,588$ and $592.2 \mathrm{GHz}$. The inset shows the normalized transmission for $0 \%$ and $100 \%$ intensity measured over the entire frequency range of 570-600 GHz. A modulation depth, or "on" and "off" ratio of $\sim 20 \mathrm{~dB}$ has been obtained [15].

Previous demonstrations have shown that free-carrier absorption in Si can be effective for optically-controlled modulation of continuous waves in the frequency range of 570-600 GHz [15]. In this work, we have extended this concept to spatially encode the array pixels for reconfigurable Hadamard aperture masks. Fig. 2 shows the measured THz responses from the detector as a function of DLP light intensity $(0 \%-100 \%, 100 \%$ corresponding to white light at 2500 lumens) for three $\mathrm{THz}$ frequencies (i.e., $578.4 \mathrm{GHz}, 588 \mathrm{GHz}$ and $592.2 \mathrm{GHz}$ ). With increasing of the photo-excitation intensity, the density of free carriers increases, resulting in 
reduced transmitted $\mathrm{THz}$ power (power is proportional to the detector output voltage in the square-law region of the detector). The inset of Fig. 2 shows the normalized $\mathrm{THz}$ transmission (normal to the response without light) for $0 \%$ and $100 \%$ intensity measured over the entire frequency range of 570-600 GHz. A modulation depth, or the "on" (with $0 \%$ or black light) and "off" (with $100 \%$ or white light) ratio of $\sim 20 \mathrm{~dB}$ at $585 \mathrm{GHz}$ has been obtained. A relatively flat modulation depth is obtained over the frequency range of 578-592 $\mathrm{GHz}$.

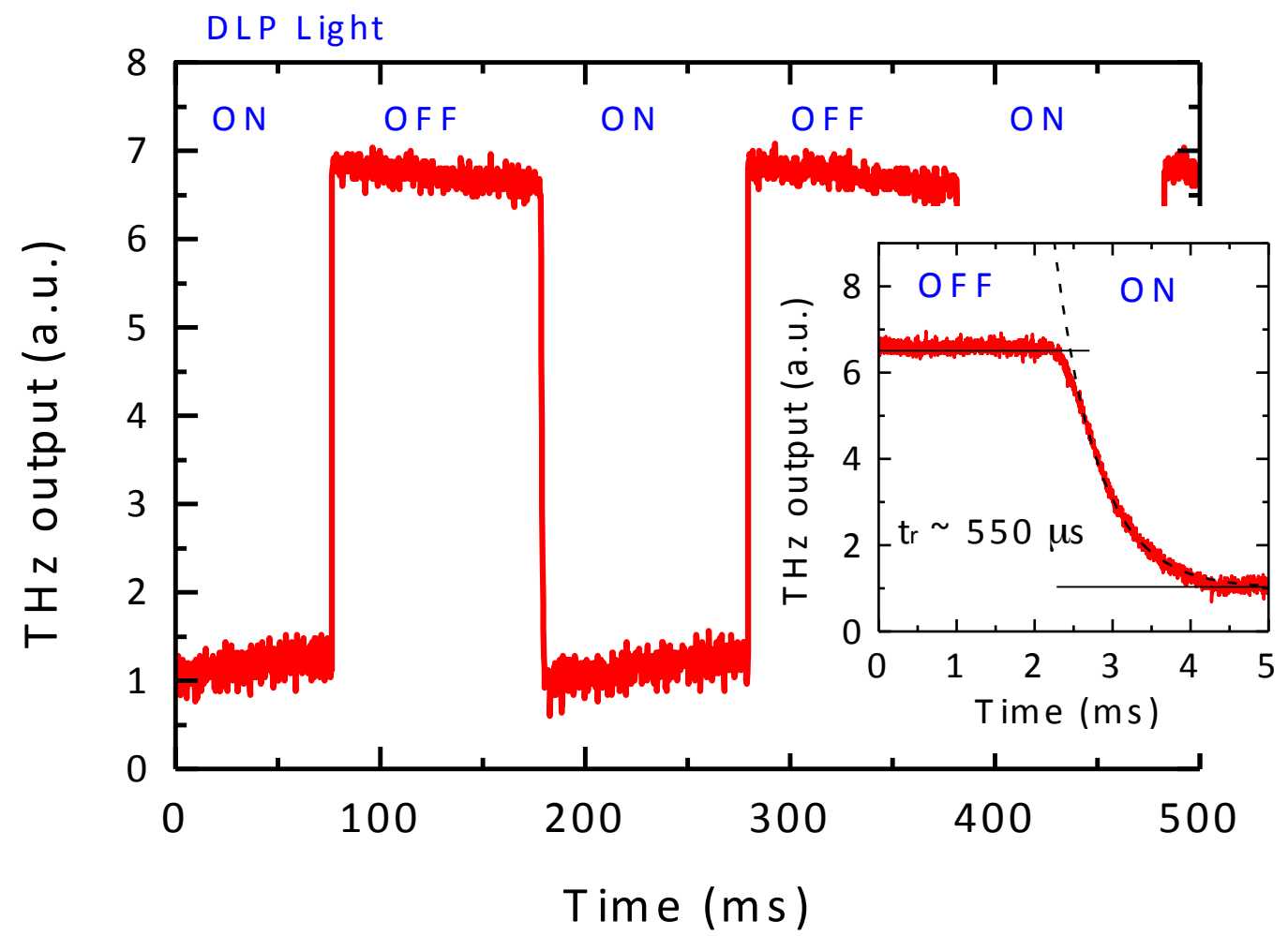

Fig. 3: Time response of $\mathrm{THz}$ wave modulation by switching DLP projection light at a rate of $5 \mathrm{~Hz}$. The inset shows the enlarged view of transition. The transition time was determined by the switching rate of DMD panel and was measured to be $\sim 550 \mu \mathrm{s}$.

To estimate the modulation speed, we illuminated uniform white light (100\%) pulses onto the silicon wafer by programming the DLP to "flash" at a frequency of $5 \mathrm{~Hz}$. Figure 3 shows the modulated $\mathrm{THz}$ output signals $(585 \mathrm{GHz})$ corresponding to the photo-excitation from the 
DLP. The transient response (Fig. 3 inset) of the temporal THz modulation shows a $10-90 \%$ rise (from "off" to "on") time of $\sim 550 \mu \mathrm{s}$, corresponding to a 3 -dB bandwidth of $\sim 1.3 \mathrm{kHz}$. This modulation speed is slower than what is expected based on the free carrier recombination rate in silicon [18], but rather could be limited by the speed of the DMD array control electronics $(\sim 1.9 \mathrm{kHz})$ in the DLP projector [19]. High speed DMD chipsets such as DLPC410 by Texas Instruments, Inc. could be employed to provide a $32 \mathrm{kHz}$ frame rate for much improved imaging speed [19].

(a)
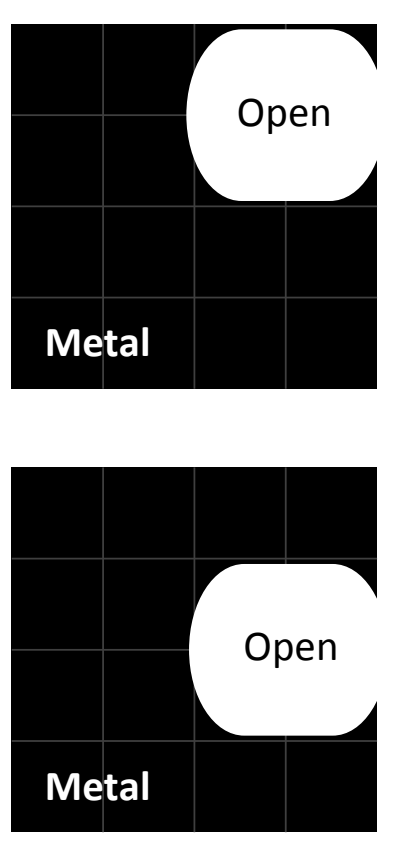

(b)
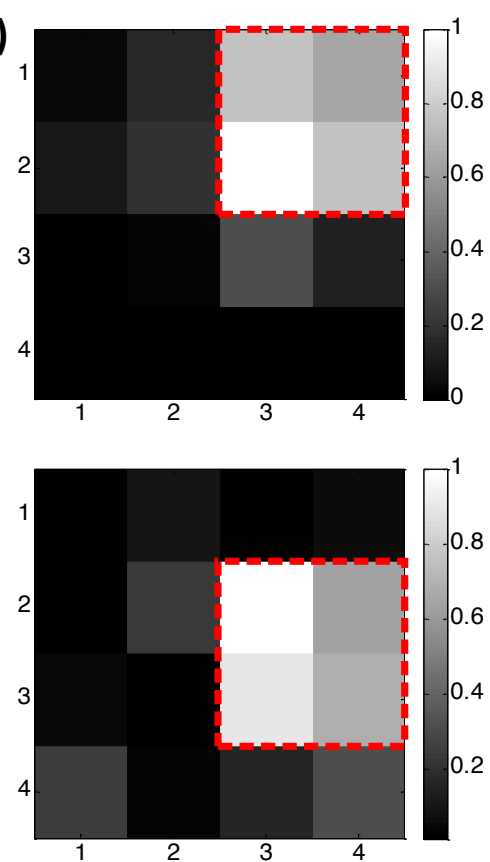

(c)
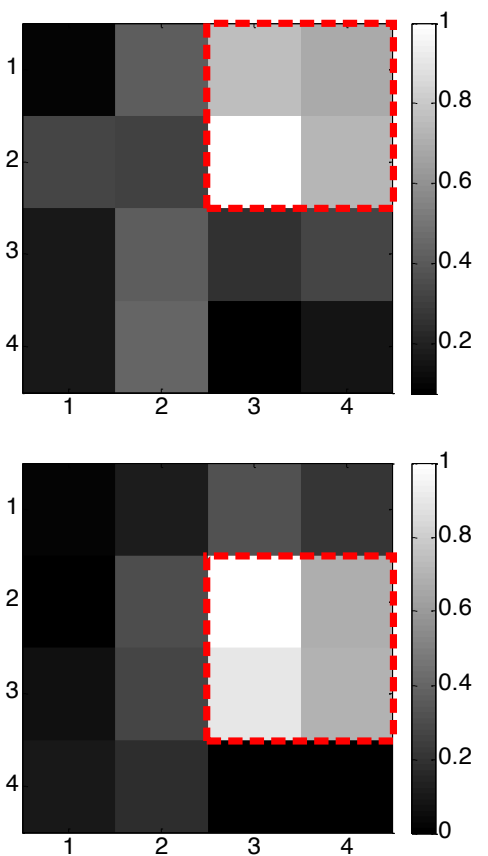

Fig. 4: 4 x $4 \mathrm{THz}$ CAI at $590 \mathrm{GHz}$ using reconfigurable photo-induced aperture arrays on a silicon wafer. (a) Left column: thin metal pieces (covered by absorber) with aperture openings at different positions as imaging objects. (b) Middle column: Single aperture CAI results. (c) Right column: Hadamard CAI imaging results.

For a prototype demonstration of $\mathrm{THz} \mathrm{CAI}$ using the photo-induced reconfigurable aperture arrays (as seen in Fig. 1), we performed THz imaging at $590 \mathrm{GHz}$ with a 4 x 4 pixel array. In this experiment, we employed the well-known Hadamard coding [11] (4 x 4 Hadamard matrix containing "1" and "-1" elements) for generating a series of coded masks. In order to 
generate the "-1" elements in the Hadamard matrix, we recorded the result corresponding to each matrix using an array with elements " 1 " (aperture “on") and "0" (aperture "off”) and its complementary array, and then subtracted the two measurements from each other. In this way, a total number of 32 arrays were used for imaging with $4 \times 4$ pixels. The use of compressed CAI [12] can significantly reduce the required number of masks. To verify the effectiveness of this imaging approach, a thin metal target (covered by absorber) with an open aperture (see Fig. 1 inset) was used as the test object.

The imaging was performed automatically with a LabView program. Each array pattern was illuminated onto the silicon wafer for one second followed by a full black image for another second to reduce the heat effect on the silicon wafer introduced by the DLP projector. The above procedure was repeated with reconfigured Hadamard patterns for 32 times. 16 linear equations were then built and solved to reconstruct the image for the object. For comparison, imaging results using the single aperture CAI as described in [15] are shown in Fig. 4(b) and shown in Fig. 4(c) are the imaging results using the Hadamard coding for two different objects. The reconstructed images agree quite well with the original objects showing this technique is promising for $\mathrm{THz}$ imaging. Since the modulation speed of this approach was estimated to be $\sim 1.3 \mathrm{kHz}$, a real-time video-rate (30 frame/sec) imaging with $7 \mathrm{x} 7$ resolution can be potentially demonstrated. A real-time low-cost $\mathrm{THz}$ camera for many practical applications could be realized on the basis of this approach.

In addition, we applied the above $\mathrm{THz}$ imaging approach to map the $\mathrm{THz}$ beam in the experimental setup as shown in Fig. 1. The THz beam waist (or radius $w$ ) size as a function of distance from the parabolic mirror M4 (d) was first calculated using Gaussian beam transformation described in [20] and the result is shown in Fig. 5 (a). For mapping the THz beam, the imaging object (thin metal piece) for the prototype CAI demonstration was 


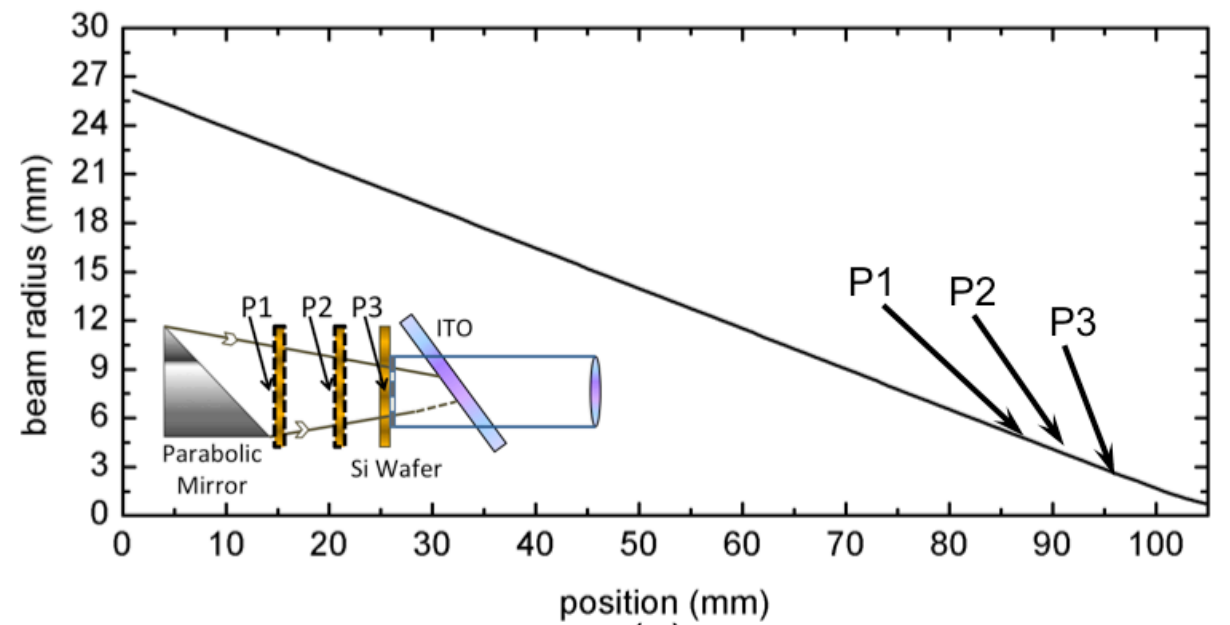

(a)
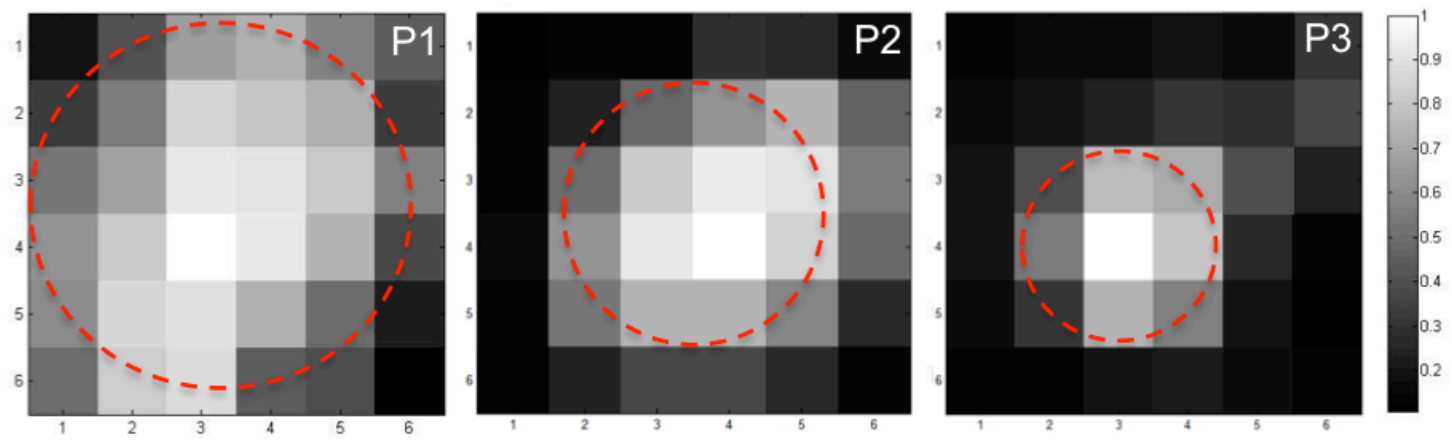

(b)

Fig. 5. (a) Calculated THz beam waist as a function of the distance from the parabolic mirror M4. The inset shows the three positions chosen for taking $\mathrm{THz}$ images using the reported $\mathrm{THz}$ CAI approach. (b) Images of the $\mathrm{THz}$ beam in the quasi-optical system (shown in Fig. 1) at the three positions indicated in (a). Red dashed circles show the calculated THz beam sizes $(\sim 9 \mathrm{~mm}, 7 \mathrm{~mm}$, and $5 \mathrm{~mm}$ for P1, P2, and P3, respectively) using Gaussian beam transformation [19]. 
removed from the system. The silicon wafer was moved to three different positions $\mathrm{P} 1\left(d_{1}=\right.$ $88 \mathrm{~mm}), \mathrm{P} 2\left(d_{2}=92 \mathrm{~mm}\right)$ and $\mathrm{P} 3\left(d_{3}=96 \mathrm{~mm}\right)$ as shown in the inset of Fig. 5(a). During this process, the projected image from the DLP was kept focused (DLP moved together with the silicon wafer) and the imaging area on the silicon wafer remained $10 \times 10 \mathrm{~mm}^{2}$. As shown in Fig. 5(b), THz images at the three positions were taken using the above approach with $6 \times 6$ pixels. Although with relatively low resolution, all three images clearly show the $\mathrm{THz}$ beam at the positions where the silicon wafer was placed with a brighter region at the imaging area center. The red dashed circles $(\sim 4.5 \mathrm{~mm}, 3.5 \mathrm{~mm}$ and $2.5 \mathrm{~mm}$ radius for P1, P2 and P3 respectively) show the calculated $\mathrm{THz}$ beam sizes based on the calculation in Fig. 5(a), indicating that good agreement between the theoretical calculation and the experiment has been obtained. This suggests that this approach is a simple but potentially powerful means to visualize $\mathrm{THz}$ beams in a quasi-optical system. This same technique could be quickly refined and applied to quantum-cascade laser optimization and characterization, as well as $\mathrm{THz}$ antenna characterization. Future work will be focused on the development of real-time and low-cost $\mathrm{THz}$ cameras with more sophisticated coding, specifically designed DLP chips/systems, and high-performance $\mathrm{THz}$ detectors and receivers $[6,7]$.

In conclusion, we report a novel approach for terahertz CAI using photo-induced reconfigurable aperture arrays on a silicon wafer illuminated by a commercially available DLP projector. At $590 \mathrm{GHz}$, each of the array element aperture can be optically turned on and off with a modulation depth of $20 \mathrm{~dB}$ and a speed of $\sim 1.3 \mathrm{kHz}$. Prototype demonstrations of 4 $\times 4$ coded-aperture imaging using the Hadamard coding have been performed and this technique has been successfully applied to mapping THz beams with $6 \times 6$ pixels at 590 GHz. The reported approach provides a simple but powerful means to visualize $\mathrm{THz}$ beams, which is highly desired in quasi-optical system alignment, quantum-cascade laser design and $\mathrm{THz}$ antenna characterization. 


\section{Acknowledgement}

This work was partially supported by NSF Grants ECCS-1002088, ECCS-1102214 and ECCS-1202452. The authors also would like to acknowledge partial supports from the Advanced Diagnostics and Therapeutics (AD\&T) and the Center for Nano Science and Technology (NDnano) at the University of Notre Dame.

\section{Reference:}

[1] B. Hu, M. Nuss, Optics Letters 20, 1761 (1995).

[2] T. G. Phillips, J. Keene, Proceedings of the IEEE 80, 1662-1678 (1992).

[3] A. Markelz, A. Roitberg, E. J. Heilweil, Chemical Physics Letters 320, $42-48$ (2000).

[4] E. Brown, D. Woolard, A. Samuels, T. Globus and B. Gelmont, IEEE MTT-S Int. Microwave Symp. Digest 3, 1591-1594 (2002).

[5] D. B. Rutledge, and M. S. Muha, IEEE Trans. Antennas Propagat. 30, 535-540 (1982).

[6] L. Liu, H. Xu, A. W. Lichtenberger, and R. M. Weikle, II, IEEE Trans. Microwave Theory Tech. 58, 1943-1951 (2010).

[7] S. Rahman, Z. Jiang, Y. Xie, H. Xing, P. Fay, L. Liu, 23th Int. Symp. Space THz Tech., Tokyo, Japan (2012).

[8] D. J. Burdette, J. Alverbro, Z. Zhang, P. Fay, Y. Ni, P. Potet, K. Sertel, G. Trichopoulos, K. Topalli, J. Volakis, H. Lee Mosbacker, SPIE proceedings 8023, (2011).

[9] M. Cui, J. N. Hovenier, Y. Ren, N. Vercruyssen, J. R. Gao et al., Appl. Phys. Lett. 102, 111113 (2013).

[10] C. H. Smith, III, H. Xu, J. L. Hesler, N. S. Barker, 33rd International Conference on Infrared, Millimeter and Terahertz Waves (IRMMW-THz), Pasadena, CA (2008).

[11] I. Valova, Y. Kosugi, IEEE Trans. Information Technology in Biomedicine 4, 306-19 (2000).

[12] W. L. Chan, K. Charan, D. Takhar, K. F. Kelly, R. G. Baraniuk, and D. M. Mittleman, Appl. Phys. Lett. 93, 121105 (2008). 
[13] S. Hawasli, N. Alijabarri, R. M. Weikle II, $37^{\text {th }}$ International Conference on Infrared, Millimeter, and Terahertz Waves, Wollongong, NSW, Australia, (2012).

[14] B. Sensale-Rodriguez, S. Rafique, R. Yan, M. Zhu, V. Protasenko, D. Jena, L. Liu, H. G. Xing, Opt. Express 21, 2324-2330 (2013).

[15] L. Cheng, L. Liu, Opt. Express, submitted (2013).

[16] L. Liu, R. Pathak, L.-J. Cheng, and T. Wang, Sensors and Actuators B 184, 228-234 (2013).

[17] L. Liu, J. L. Hesler, H. Xu, A. W. Lichtenberger, R. M. Weikle, II, IEEE Microwave and Wireless Components Letters 20, 504-506 (2010).

[18] H. Alius, G. Dodel, Infrared Phys. 32, 1-11 (1991).

[19] http://www.ti.com/lit/ds/symlink/dlpc410.pdf

[20] P. F. Goldsmith, "Quasioptical systems: Gaussian beam quasi-optical propagation and applications," Wiley \& Sons, Inc. (1997). 